\title{
Diabetes mellitus tipo 2 no desencadeamento da Doença de Alzheimer
}

\author{
Type 2 diabetes mellitus in triggering Alzheimer's disease
}

La diabetes mellitus tipo 2 como desencadenante de la enfermedad de Alzheimer

Alexsander Augusto da Silveira ${ }^{1 *}$ Aline da Silva Nunes ${ }^{1}$, lânica Neves Correia Sevilha ${ }^{1}$, Ruth Thalita da Silva Nunes ${ }^{1}$, Álvaro Paulo Silva Souza ${ }^{1}$, Adibe Georges Khouri' ${ }^{1}$, Mariana Cristina de Morais ${ }^{1}$, Lucas Nojosa Oliveira ${ }^{1}$, Adeliane Castro da Costa ${ }^{1}$.

\section{RESUMO}

Objetivo: Descrever a relação da resistência periférica dos receptores para a insulina como promotora e prognóstico no desenvolvimento da Doença de Alzheimer (DA), assim como as novas tendências medicamentosas profiláticas as quais interrompem a progressão da DA. Revisão Bibliográfica: A insulina desempenha importantes funções como fator de crescimento e regulando o metabolismo da glicose, ácidos graxos e proteínas. Fatores como obesidade, inatividade física e dieta contendo alto teor de gordura e açúcar estão fortemente associados à resistência à insulina e ao diabetes mellitus do tipo 2. No cérebro, a insulina atua no crescimento e diferenciação dos neurônios, participa das funções cognitivas superiores, na formação de memória de longo prazo e tem efeito neuroprotetor. Considerações Finais: A hiperglicemia, peptídeos sinalizadores pró inflamatórios, o estresse oxidativo e a sinalização interrompida da insulina cerebral estão associados com a promoção ao desenvolvimento e a progressão da DA. A insulina pode ser efetivamente entregue diretamente ao cérebro por meio da via intranasal que permite ao hormônio ultrapassar a barreira hematoencefálica e modular as funções nervosas centrais.

Palavras-chave: Inflamação, Estresse oxidativo, Resistência à insulina, Demência.

\begin{abstract}
Objective: To describe the relationship of peripheral resistance of receptors to insulin as a promoter and prognosis in the development of Alzheimer's Disease (AD), as well as the new prophylactic drug trends that interrupt the progression of $A D$. Bibliographic Review: Insulin plays an important role as a growth factor and regulates the metabolism of glucose, fatty acids and proteins. Factors such as obesity, physical inactivity and a diet with a high fat and sugar content are strongly associated with insulin resistance and type 2 diabetes mellitus. In the brain, insulin acts in the growth and differentiation of neurons, participates in higher cognitive functions, in formation of long-term memory and has a neuroprotective effect. Final Considerations: Hyperglycemia, pro-inflammatory signal peptides, oxidative stress and interrupted brain insulin signaling are associated with the promotion and development and progression of $A D$. Insulin can be effectively delivered directly to the brain via the intranasal route that allows the hormone to overcome the blood-brain barrier and modulate central nerve functions.
\end{abstract}

Keywords: Inflammation, Oxidative stress, Insulin resistance, Dementia.

\section{RESUMEN}

Objetivo: Describir la relación de la resistencia periférica de los receptores a la insulina como promotor y pronóstico en el desarrollo de la Enfermedad de Alzheimer (EA), así como las nuevas tendencias de fármacos profilácticos que interrumpen la progresión de la EA. Revisión bibliográfica: La insulina juega un papel importante como factor de crecimiento y regula el metabolismo de la glucosa, los ácidos grasos y las proteínas. Factores como la obesidad, la inactividad física y una dieta con alto contenido de grasas y azúcares están fuertemente asociados con la resistencia a la insulina y la diabetes mellitus tipo 2. En el cerebro, la insulina actúa en el crecimiento y diferenciación de las neuronas, participa en funciones cognitivas superiores, en formación de memoria a largo plazo y tiene un efecto neuroprotector. Consideraciones finales: La hiperglucemia, los péptidos señal proinflamatorios, el estrés oxidativo y la señalización cerebral interrumpida de la insulina están asociados con la promoción, el desarrollo y la progresión de la EA. La insulina se puede administrar de manera efectiva directamente al cerebro a través de la ruta intranasal que permite que la hormona supere la barrera hematoencefálica y module las funciones del nervio central.

Palabras clave: Inflamación, Estrés oxidativo, Resistencia a la insulina, Demencia.

${ }^{1}$ Faculdade Estácio de Sá de Goiás, Goiânia - GO. *E-mail: alekfarm2000@yahoo.com.br 


\section{INTRODUÇÃO}

A insulina é um hormônio proteico que regula a homeostase de glicose, ativando a glicólise, lipogênese e glicogênese, induzindo efeitos hipoglicemiantes. O estímulo gerado pela insulina recruta transportadores de glicose (GLUT) para a membrana celular garantindo o transporte da glicose para o interior da célula (KLIP A, et al., 2019). No cérebro, controla funções fisiológicas cerebrais assegurando a plasticidade neuronal e a oferta energética, no entanto a captação de glicose no cérebro não é dependente de insulina (TAOUIS M e TORRES-ALEMAN I, 2019). As desordens metabólicas provocadas neste órgão estão associadas ao desenvolvimento de doenças neurodegenerativas, incluindo a Doença de Alzheimer (DA) (ASMAT U, et al., 2016).

Durante o desenvolvimento da obesidade e diabetes mellitus (DM) é estabelecido a indução da resistência à insulina, com a diminuição na oferta de glicose nos tecidos. A DM é um distúrbio metabólico crônico caracterizada pelo aumento de glicose no sangue, conhecido como hiperglicemia, a qual pode ser devido à i) ausência ou insuficiência na produção de insulina, ou ii) pela baixa expressão de receptores periféricos para insulina nas células dos tecidos (ASMAT U, et al., 2016; OLIVEIRA JEP, et al., 2017; RAK K e BRONKOWSKA $M, 2018)$. A classificação em DM1 ocorre quando possui natureza autoimune, com destruição das células $\beta$ pancreáticas e incapacidade de produção da insulina, podendo ser induzidos por fatores genéticos ou ambientais (DIMEGLIO LA, et al., 2018; NOBLE JA, 2015; THOMAS NJ, et al., 2018).

Ao contrário, a DM2 é uma doença crônica relacionada à idade e ao estilo de vida, caracterizada pelo aumento dos níveis de glicose e insulina no sangue, resistência à insulina, anormalidades metabólicas e inflamação crônica (OLIVEIRA JEP, et al., 2017). Após a ingestão de carboidratos, é desencadeada a produção de insulina pelo pâncreas, que induz a absorção de glicose do sangue para o interior das células mediante o aumento de expressão de receptores de glicose (GLUT) nas membranas das células, com ativação de cascatas intracelulares como glicólise, lipogênese e a glicogênese (MAGALHÃES FG, et al., 2019). O distúrbio metabólico resultante leva à hiperglicemia crônica, que é a causa imediata de muitos dos sintomas do DM2, com sequenciais processos inflamatórios nos locais de depósito de glicose, os quais levam a retinopatia, neuropatia periférica e nefropatia (FRANKL JA, et al., 2016; GABBOUJ S, et al., 2019; KANDIMALLA R, et al., 2017; OLIVEIRA JEP, et al., 2017).

A DA é uma doença neurodegenerativa caracterizada pelo declínio progressivo da memória, funções cognitivas e mudanças comportamentais (PUGAZHENTHI S, et al., 2017). A DA é provocada por fatores genéticos e ambientais, podendo se agravar com a idade, histórico familiar de demência, traumatismo craniano grave, estimulação intelectual baixa, hormônios femininos, depressão prévia, dentre outros (KANDIMALLA R, et al., 2017; MONTEIRO AMF, et al., 2018). A sintomatologia típica da DA é o comprometimento da função cognitiva, perda de memória, problemas de linguagem, seguida por distúrbios comportamentais como agitação, agressividade e depressão (NAZARETH AM, 2017). Estudos demonstram que $\mathrm{o}$ aumento de incidência da DA está relacionado à DM2, sugerindo que a DA seja uma das complicações do DM2 (KANDIMALLA R, et al., 2017; PUGAZHENTHI S, et al., 2017).

Visto que a comorbidade entre DA e DM2 é um agravante para o prognóstico de ambas doenças, o objetivo deste artigo é demonstrar por meio de uma revisão narrativa os mecanismos moleculares relacionados entre DM2 e DA. Além disso, discorrer sobre as novas tendências medicamentosas para a DM2 que auxiliam na profilaxia, e que possivelmente interrompem a progressão da DA.

\section{REVISÃO BIBLIOGRÁFICA}

\section{Diabetes Mellitus Tipo-2 e a Doença de Alzheimer}

A diminuição na oferta de glicose ao tecido nervoso promove uma diminuição expressiva da produção de energia pelos neurônios e nas funções cognitivas (KANDIMALLA R, et al., 2017; TAOUIS M e TORRESALEMAN I, 2019). Evidências epidemiológicas sugerem uma associação entre o DM2 e DA sendo que os mecanismos de associação entre elas estão interligados por meio do processo central de resistência à insulina (STRACHAN MW, et al., 1997; KANDIMALLA R, et al., 2017). 
A DM2 é uma doença crônica em que o paciente desenvolve resistente à insulina, com possui elevados níveis de glicose no sangue, devido à baixa expressão dos receptores de insulina (IR), receptor de IGF-1 (IGF-1R), e as proteínas do substrato do receptor de insulina (IRS), promovendo a resistência periférica à insulina. Os hormônios adiponectina e pectina em indivíduos idosos agravam a doença (OLIVEIRA JEP, et al., 2017; CHATTERJEE S e MUDHER A, 2018; RINALDI G, et al., 2019; BUTTERFIELD DA, et al., 2014; RINALDI G, et al., 2019). Estes associados resultam na característica patológica de resistência à insulina nos músculos, fígado e células adiposas (CHATTERJEE S e MUDHER A, 2018).

A DA é um distúrbio neurodegenerativo progressivo associado à atrofia cerebral generalizada que se inicia no córtex transentorinal e entorrinal nos estágios iniciais e depois se espalha para as áreas neocorticais. $O$ neocórtex e o hipocampo parecem ser as áreas mais afetadas pela doença e a perda de neurônios nessas áreas é responsável por sua atrofia, inerente à disfunção cognitiva, principalmente da memória, e ao diagnóstico da doença. Além dessas áreas, os núcleos subcorticais que conectam o córtex também são afetados, incluindo o núcleo colinérgico basal de Meynert e o septo medial (NAZARETH AM, 2017).

A causa de toda esta neurodegeneração pode ser explicada por processos inflamatórios neuronais, devido ao acúmulo e formação de placas $\beta$-amilóides e emaranhados de proteínas TAU hiperfosforiladas e glicosilados nos neurônios, com consequente disfunção colinérgica. Exames histopatológicos de cérebros pós-morte revelam a presença de placas neuríticas extracelulares, emaranhados neurofibrilares intracelulares e perda neuronal. A DA também está associada à perda de sinapses, estresse oxidativo e anormalidades funcionais e estruturais das mitocôndrias, respostas inflamatórias, alterações na neurotransmissão colinérgica e hormonais, bem como anormalidades do ciclo celular neuronal (CHATTERJEE S e MUDHER A, 2018; KANDIMALLA R, et al., 2017; NAZARETH AM, 2017).

\section{Mediadores Inflamatórios Periféricos Contribuem para a Inflamação Cerebral e para Resistência à Insulina Neuronal}

A hiperglicemia crônica promove a liberação de mediadores inflamatórios e glicosilação proteica, com autooxidação das partículas de glicose, desencadeando a formação de radicais livres, destruição e disfunção celular. Consequentemente, há uma importante mudança sistêmica, que não pode ser compensada pelo sistema antioxidante, encarregado de eliminar os radicais livres do corpo (GOMES BF e ACCARDO CM, 2019).

A presença de citocinas contribuem para a inflamação cerebral, resistência à insulina neuronal e disfunção neuronal na DA. O envelhecimento por inflamação, metainflamação e infecção/inflamação periférica, causada por patógenos ou distúrbios inflamatórios sistêmicos dão origem a estados de inflamação sistêmica de baixo grau, levando à superprodução de citocinas / Interleucinas (IL) pró-inflamatórias, como Tumor Necrosis Factor - alpha (TNF- $\alpha$ ), IL-1 $\beta$ e IL-6. Níveis elevados de adipocinas (proteínas sintetizadas e secretadas pelo tecido adiposo) também podem vincular a inflamação do sistema nervoso central (SNC) na obesidade. Estes mediadores inflamatórios periféricos atravessam a barreira hematoencefálica e em conjunto com mediadores produzidos pela microglia (Macrófago do SNC) e pelos adipócitos ativados, desencadeiam a inflamação do SNC (FERREIRA ST, et al., 2014; NAZARETH AM, 2017).

A associação clínica entre DM2 e DA levou à hipótese de que moléculas pró-inflamatórias derivadas da periferia também poderiam influenciar na patogênese no SNC. A resistência à insulina parece ocorrer por fatores genéticos ou falha no reconhecimento do hormônio pelo IR IR devido a um aumento nos níveis de ácidos graxos, glicerol e glicose. A glicose oxidada e glicosilada causa severa lesão tecidual, o que estimula o processo inflamatório no SNC.

Uma vez ativado o processo inflamatório tecidual, as células inflamatórias residentes como macrófagos e células dendríticas (micróglia) passam a sintetizar e secretar citocinas pró- inflamatórias como como IL-1 $\beta$, IL-6 e TNF- $\alpha$. Nos neurônios, essas citocinas promovem a ativação de moléculas como $c$-Jun $N$-Terminal Kinase (JNK) levando à fosforilação de serina do receptor IRS-1, inibindo a fosforilação de tirosina e desencadeando resistência à insulina (Figura 1) (FELICE FG et al., 2014; FERREIRA ST et al., 2014; NAZARETH AM, 2017; NETH BJ e CRAFT S, 2017). 
As citocinas pró-inflamatórias estimulam as vias intracelulares amiloidogênicas (APP) nas células do SNC, promovendo um aumento nos níveis de oligômeros da proteína $\beta$-amilóide $(\alpha \beta)$ característico da DA (NETH BJ e CRAFT S, 2017). A supra regulação de citocinas pró-inflamatórias medeiam a estimulação da produção de APP e a via amiloidogênica, induzindo a produção de peptídeos $\alpha \beta$. Portanto, a ativação da resposta imune inata e a produção de $\alpha \beta$ colaboram para a disfunção e morte dos neurônios, uma vez que o acúmulo de $\alpha \beta$ juntamente com a inflamação induz estresse oxidativo e diminui a sinalização da insulina no cérebro (BUTTERFIELD DA, et al., 2014; FELICE FG, et al., 2014; NETH BJ e CRAFT S, 2017).

Em adição, a ativação de receptores de citocinas neuronais, como por exemplo, receptor de TNF- $\alpha$ induz uma ativação aberrante das quinases de estresse (c-Jun N-terminal [JNK], IKBa cinase [IKK] e proteína cinase dependente de RNA de cadeia dupla [PKR]), que fosforilam o substrato 1 do receptor de insulina (IRS-1) nos resíduos de serina e inibem a fosforilação fisiológica da tirosina induzida por insulina do IRS-1. Isso interfere na capacidade do IRS-1 de se envolver na sinalização da insulina e bloqueia as ações intracelulares da insulina (FELICE FG, et al., 2014; FERREIRA ST, et al., 2014). A Figura 1 apresenta as citocinas próinflamatórias na infraregulação dos IRSs (FERREIRA ST, et al., 2014).

Figura 1 - Processo inflamatório causado pela DM2 e o dano no SNC.

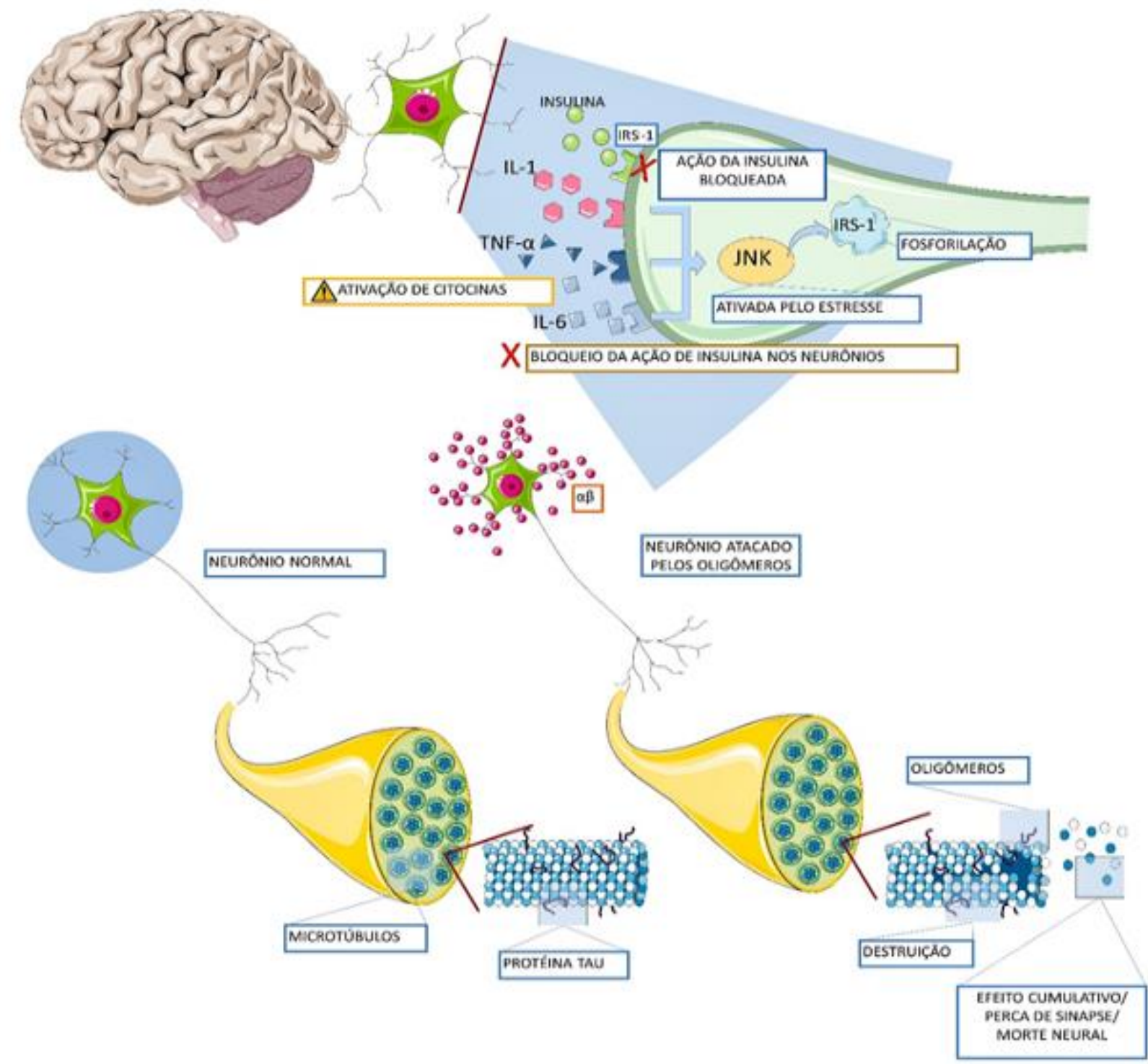

Legenda: Participação das citocinas pró-inflamatórias na infraregulação dos receptores para insulina (IRS) no SNC e na desestruturação da proteína TAU, com consequente agregação da proteína amiloidal nos neurônios. A $\mathrm{B}$ : proteína beta amiloide; JNK: quinases de estresse c-Jun N-terminal; IL-1: interleucina 1; II-6: interleucina 6; TNF- $\alpha$ : fator de necrose tumoral-a; IRS: receptor para insulina. Fonte: ilveira AA, et al., 2020. Baseado em: Ferreira ST, et al., 2014; Felice FG, et al., 2014. 
Evidências mostraram que a deficiência e resistência à insulina, e os marcadores do DM2 favorecem patologia da DA. Os IRSs são expressos nos sistemas periféricos, assim como no SNC, especialmente no hipocampo, primeira estrutura afetada na DA. A ligação da insulina a IRS leva à fosforilação da tirosina quinase e à ativação do substrato do receptor do IRS, que ativa a fosfatidilinositol-3 quinase (PI3K) e Protein kinase $B$ (Akt). A Akt medeia a fosforilação ou inativação do glicogênio sintase quinase $3 \beta$ (GSK3 $\beta$ ) na cascata da glicólise e na geração de energia. Uma vez que a sinalização intracelular para a insulina se encontra prejudicada, resulta em aumento da GSK3 $\beta$, o que faz com que a formação de emaranhados neurofibrilares, a hiperfosforilação da proteína TAU nos neurônios, aumentando a produção de $\alpha \beta$.

No encéfalo durante a DA, os oligômeros $\alpha \beta$ levam à ativação anormal do TNF- $\alpha$ via c-Jun-quinase Nterminal (JNK), inibindo IRS1 e interrompendo a sinalização pelo hormônio insulina. Outro ponto a ser considerado é participação da enzima degradadora de insulina (IDE) responsável pela degradação da via APP e da eliminação natural da proteína $\alpha \beta$. Sob condições de resistência à insulina, há competição entre insulina e $\alpha \beta$ pelos pontos de clivagem pelo IDE, que eventualmente reduz a degradação de $\alpha \beta$ o que proporciona o seu acúmulo e deposição nos neurônios (LI R, et al., 2019).

\section{Diminuição de Receptores para Insulina e Estresse Oxidativo no SNC}

Como visto acima, a neuroinflamação é uma característica que está envolvida na fisiopatologia da DA (Figura 1). $O$ acúmulo da proteína $\alpha \beta$ juntamente com a presença do excesso de glicose induzem o estresse oxidativo, com formação de radicais ou intermediários reativos do oxigênio (ROls) a partir do oxigênio molecular (O2), tais como o ânion superóxido (O2-), peróxido de hidrogênio ( $\mathrm{H} 2 \mathrm{O} 2)$, óxido nítrico (NO), peróxido nitrito (ONOO-) e ácido hipocloroso $(\mathrm{HOCl})$, o que diminui a sinalização da insulina no cérebro (NAZARETH AM, 2017; NETH BJ e CRAFT S, 2017) (Figura 2).

A resistência à insulina e a produção de $\alpha \beta$ passam a ser consideradas as principais causas do aumento do estresse oxidativo. Este último promove a fosforilação do IRS-1/2 Ser-312, -616 e -636, o qual gera negativamente impactos nos dois principais braços da cascata de sinalização mediada por insulina, com as vias intracelulares da Proteína Fosfatidilinositol 3 Quinase (PI3K) e da mitogênio quinases de ativação neuronal (MAPK). Ambas as cascatas estão envolvidas na manutenção da plasticidade sináptica e na resposta ao estresse celular (BUTTERFIELD DA, et al., 2014).

Adicionalmente, o dano oxidativo atinge as proteínas envolvidas na glicólise, ciclo de Krebs e síntese de Adenosina Trifosfato (ATP) que são eventos cruciais na redução do metabolismo da glicose (BUTTERFIELD DA, et al., 2014; FERREIRA ST, et al., 2014) (Figura 2D). As espécies reativas de oxigênio geradas por essas vias aceleram o processo de morte neuronal. Ao mesmo tempo, a resistência à insulina prejudica as vias de sinalização a jusante e exacerba a formação de oligômeros $\alpha \beta$ e agregados de TAU hiperfosforilada. $O$ efeito cumulativo de todos esses fatores expõe os neurônios a uma série de ataques e gradualmente resulta na perda de sinapses e morte neuronal (Figuras 1 e $\mathbf{2 C}$ e D). A DM2 e a DA compartilham muitas características, incluindo inflamação crônica, estresse oxidativo, sinalização de insulina prejudicada, resistência à insulina, intolerância à glicose e comprometimento cognitivo (CHATTERJEE S e MUDHER A, 2018; LI R, et al., 2019) (Figura 2A e B).

Finalmente, como a resistência à insulina está associada ao aumento da produção de $\alpha \beta$, e a produção de $\alpha \beta$ é responsável pelo início da resistência à insulina, resta esclarecer se este evento é uma causa, consequência ou resposta compensatória à neurodegeneração induzida por $\alpha \beta$. A desativação da sinalização da insulina resulta em transporte de glicose prejudicado, com a translocação reduzida do transportador de glicose na membrana plasmática (GLUT) e no metabolismo.

Este processo promove uma alteração dos processos mitocondriais envolvidos na produção de energia. Os comprometimentos associados à resistência à insulina na captação e utilização de glicose estão associados ao aumento do estresse no retículo endoplasmático (REL), que desregula o metabolismo lipídico, causando acúmulo de lipídios tóxicos no cérebro. Por sua vez, o comprometimento das funções das mitocôndrias leva a um círculo vicioso no qual a produção reduzida de energia e a um aumento das espécies reativas de oxigênio e nitrogênio (ROIs) responsáveis pelo dano oxidativo das mitocôndrias e de outros componentes celulares. Além disso, o aumento da produção e acumulação de $\alpha \beta$, que representa uma característica fundamental da patologia da DA, também promove comprometimento mitocondrial. De forma 
conjunta, todos esses eventos contribuem para o aumento dos níveis de estresse oxidativo responsáveis pela neurodegeneração observada no cérebro com DA (BUTTERFIELD DA, et al., 2014; FERREIRA ST, et al., 2014) (Figura 2C e D).

Figura 2 - Processos inflamatórios e formação dos intermediários reativos do oxigênio (ROIs) relacionados à neurodegeneração na doença de Alzheimer.

A)

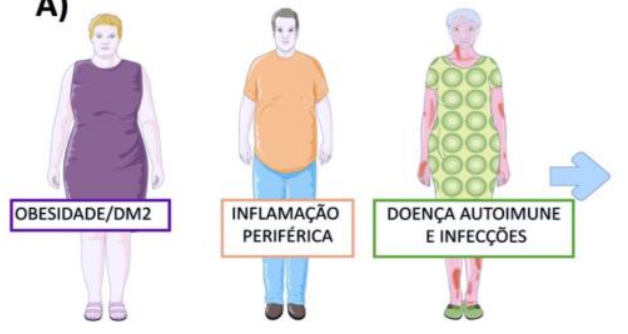

D)

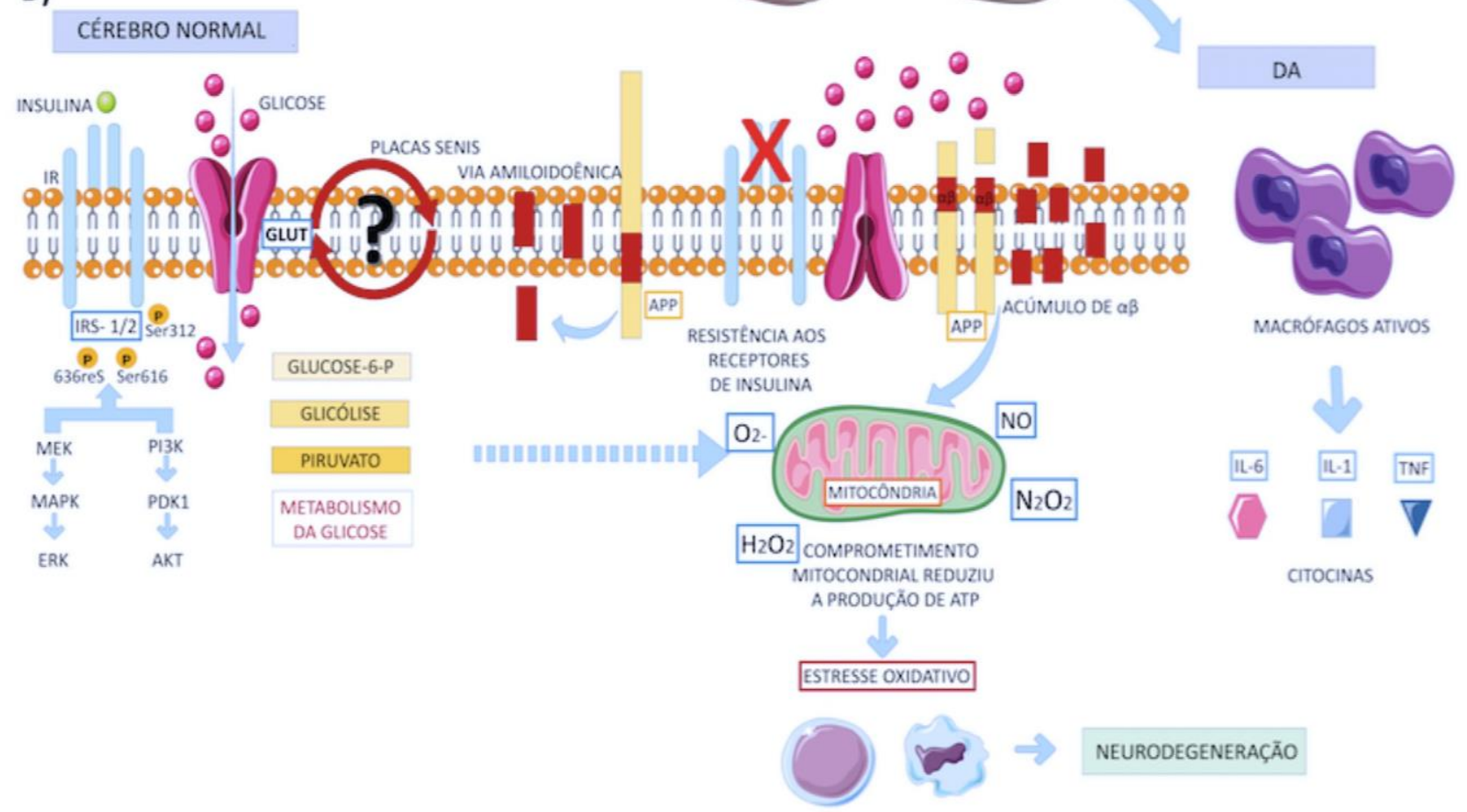

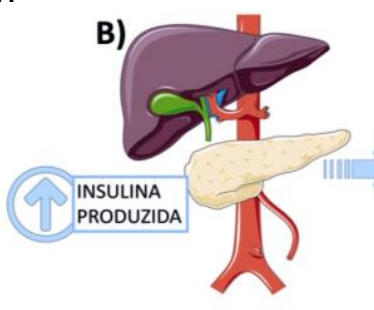
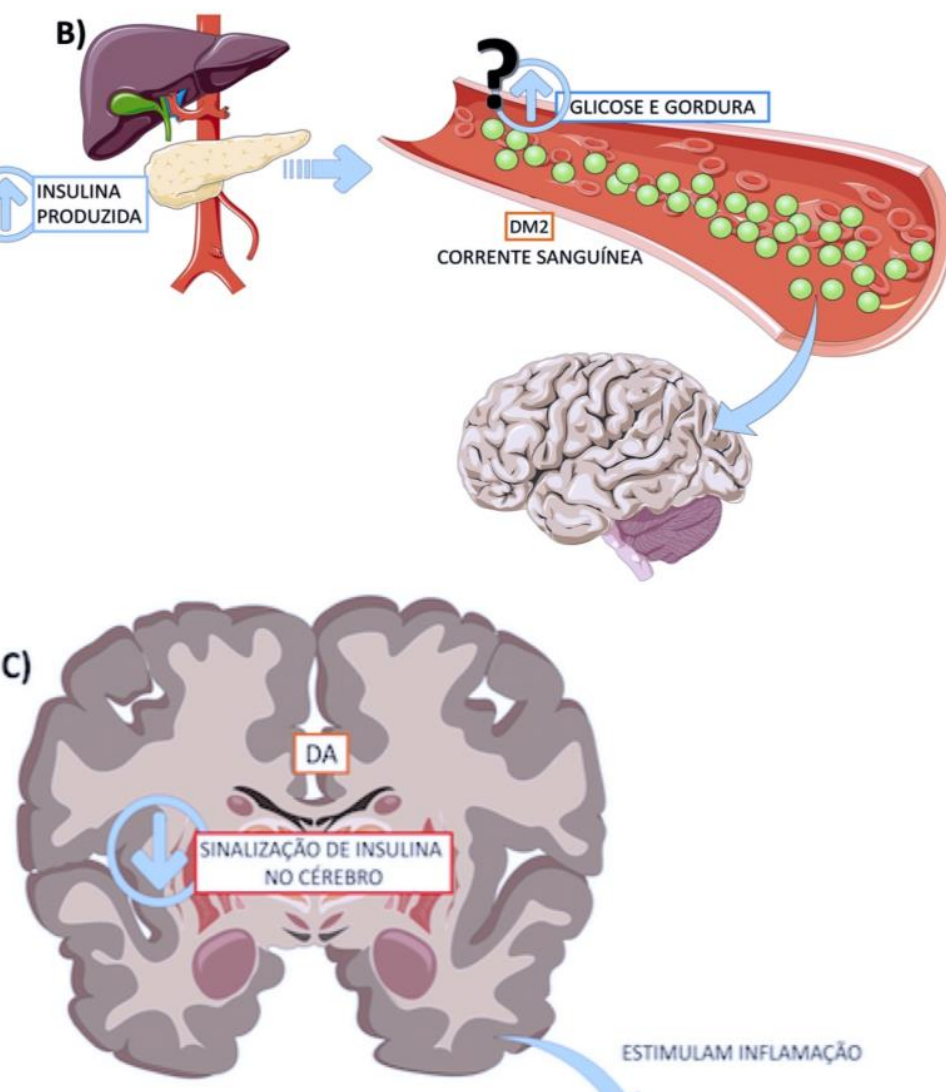

ESTIMULAM INFLAMAÇÃO

Legenda: DM2: diabetes mellitus do tipo 2; IRS: receptor para insulina; GLUT: receptor para glicose; DA: Doença de Alzheimer; APP: via intracelular amiloidogênica; O2-, H2O2, NO, N2O2: intermediários reativos do oxigênio; IL-1, IL-6 e TNF: citocinas pró - inflamatórias. Fonte: Silveira AA, et al., 2021. Baseado em: Butterfield DA, et al., 2014; Ferreira ST, et al., 2014; Chatterjee S e Mudher A, 2018; Li R, et al., 2019. 


\section{Novos Tratamentos versus Bases Moleculares: DA e DM2}

Tem sido demonstrado que há um aumento da captação de glicose no tecido da medula espinhal, em várias regiões do cérebro, como o plexo coróide, glândula pineal e hipófise. A ação da insulina no cérebro desempenha numerosos papéis críticos no controle das funções fisiológicas vitais como homeostase energética, plasticidade neuronal e metabolismo do crescimento, lipídios e glicose. Além disso, a alteração da ação da insulina observada no estado resistente à insulina ou obesidade é considerada um fator de risco para diversas patologias, incluindo doenças neurodegenerativas e distúrbios metabólicos (SURKOVA EV, et al., 2019; TAOUIS M e TORRES-ALEMAN I, 2019).

Estudos indicam a ampla distribuição de IRSs no cérebro, particularmente no bulbo olfativo, córtex, hipocampo e hipotálamo, indicando um emaranhado e complexo papel neurorregulador induzido pela insulina. Os IRSs no cérebro estão amplamente expressos em neurônios em comparação com a glia e concentrados nas regiões de sinapse neuronais. No SNC os IRSs desempenham diversas funções, incluindo regulação homeostática, modulação da plasticidade sináptica, neurotransmissão e neurodegeneração relacionada à idade (CHATTERJEE S e MUDHER A, 2018).

Sabendo que a DA é um fator de risco para a DM2, e vice-versa, estratégias terapêuticas para superar a deficiência e a resistência à insulina no SNC podem ser uma opção no tratamento de deficiências cognitivas como a DA. A insulina pode ser efetivamente entregue diretamente ao cérebro por meio da via intranasal que permite o hormônio ultrapassar a barreira hematoencefálica e modular as funções nervosas centrais (BENEDICT C, et al., 2011; VIEIRA MNN, et al., 2018).

A captação de glicose nos tecidos periféricos é baseada no transportador de glicose dependente de insulina 4 (GLUT4). A insulina ativa a via PI3K-Akt e a Akt quinase ativada posteriormente fosforila o substrato Akt de $160 \mathrm{kDa}$ (AS160), que recruta GLUT4 para a membrana plasmática, permitindo que a glicose entre com eficiência na célula. No cérebro, as células endoteliais e os astrócitos, componentes da barreira hematoencefálica, expressam principalmente GLUT1, enquanto o transportador de glicose mais comum nos neurônios é o GLUT3. Os transportadores de glicose GLUT1 e GLUT3 são independentes de insulina. No entanto, foi demonstrado que o GLUT4 dependente de insulina é expresso em certa medida em várias regiões do cérebro, como hipocampo, cerebelo e bulbo olfativo (GABBOUJ S, et al., 2019).

A sinalização da insulina no cérebro desempenha um papel crítico no controle metabólico e na função cognitiva. O direcionamento das vias insulinérgicas no SNC por meio da administração periférica de insulina é possível, mas associado a efeitos sistêmicos que requerem supervisão rigorosa ou contramedidas (SANTIAGO JCP e HALLSCHMID M, 2019).

A terapia combinada pode levar a melhores resultados para pacientes com resistência à insulina. Os antioxidantes prometem tratamentos eficazes para o estresse oxidativo. $O$ ácido ascórbico e os tocoferóis foram bem-sucedidos na reabilitação de tecidos periféricos e aumento da sensibilidade à insulina. Os flavonoides e o ácido $\alpha$-lipóico mostraram qualidades semelhantes. O futuro uso de antioxidantes pode chegar à prática clínica com maior compreensão dos mecanismos que eles empregam. Uma dieta variada e nutritiva pode ser empregada como medida preventiva para a saúde metabólica e como um complemento ao tratamento metabólico. A manutenção de um peso saudável também é importante para o tratamento da resistência à insulina (HURRLE S e HSU WH, 2017).

A terapia com injeção de insulina é difícil e dolorosa para muitos pacientes, portanto, as rotas mais recentes para a administração de insulina são a direção atual da pesquisa de insulina, incluindo a administração por inalação. Estudos em animais demonstraram que a insulina pode ser transferida, sem comprometer suas propriedades biológicas, ao longo das vias olfativas e trigeminais para o cérebro por via nasal. O nervo olfativo termina no bulbo olfativo, enquanto o nervo trigêmeo entra no cérebro através dos pontos da placa cribriforme, permitindo a administração de drogas nas regiões anterior e posterior do cérebro. Sugere-se que o transporte de substâncias ao longo das vias nervosas olfativas e trigêmeos ocorra por meio de mecanismos intracelulares e extracelulares. A administração intranasal parece aumentar os níveis de insulina no cérebro em humanos, tendo a hipótese de explorar as vias do nariz para o cérebro e fornecer drogas diretamente ao tecido cerebral, limitando a exposição sistêmica (CHAPMAN CD, et al., 2018; MEHTA RJ, et al., 2019; WINGROVE J, et al., 2019). 
Desta maneira, uma terapia alternativa para corrigir a sinalização de insulina para DA é a administração de insulina intranasal (IN), a qual melhora a memória em adultos normais, previne o declínio cognitivo, atrofia cerebral, acúmulo de $\alpha \beta$ e lesões de substância branca no modelo de encefalopatia por DM1. Em um modelo de rato com DM2, a IN mostrou-se eficaz para a redução da hiperfosforilação da TAU (CHEN Z e ZHONG C, 2013).

A IN é neuroprotetora em modelos para a DA, doença de Parkinson e lesão cerebral traumática, com efeitos cognitivos benéficos para estes pacientes. Sabe-se que a insulina pode ser detectada no SNC poucos minutos após a administração IN. As vantagens da administração do medicamento IN, em relação a outras vias de administração, incluem não invasividade, facilidade de autoadministração, rápida absorção e início da ação e prevenção da eliminação hepática da primeira passagem. Devido à sua baixa biodisponibilidade ( 38\% em comparação com uma injeção intravenosa), é improvável que os níveis plasmáticos de insulina administrada por via IN causem efeitos colaterais sistêmicos, como hipoglicemia.

Além disso, a administração via IN contorna a barreira hematoencefálica para atingir o cérebro por vias diretas que se conectam a lâmina nasal própria ao SNC (GABBOUJ S, et al., 2019; LOCHHEAD JJ, et al., 2019). A aplicação intranasal contornando a barreira hematoencefálica resulta em elevação não fisiológica dos níveis centrais de insulina que podem evocar efeitos opostos na sensibilidade olfativa. No entanto, é importante observar que a insulina IN não tem como alvo específico o bulbo olfativo, mas também pode causar alterações de atividade em outros componentes do sistema olfativo, como o epitélio olfativo e o córtex olfativo. Inflamação nasal pela aplicação de insulina é descrito, o que tem que ser levado em consideração para utilização por períodos prolongados, assim como a busca por formulações farmacêuticas que contornem estes efeitos colaterais esperados (THANARAJAH SE, et al., 2019).

\section{CONSIDERAÇÕES FINAIS}

Este artigo de revisão apresenta as bases moleculares e sinalização intracelular envolvidas em duas importantes doenças degenerativas crônicas, a DM2 e DA. Este estudo também é de extrema importância para a compreensão da relação entre as duas patologias. Outro ponto imprescindível é que o entendimento molecular apresentado pode favorecer e permitir o desenho de novos fármacos, ou estabelecer novos tratamentos que possam retardar ou bloquear a progressão da doença, com aumento da sobrevida dos pacientes, com a melhora na sua qualidade de vida e benefícios para a saúde pública frente a estas doenças.

\section{AGRADECIMENTOS E FINANCIAMENTO}

Este trabalho foi realizado na Faculdade Estácio de Sá de Goiás, apoiado pela coordenação de Pesquisa e Extensão e Departamentos de Farmácia e Biomedicina. Os autores AAS ACC, MCM e LNO recebem Bolsa de Produtividade em Pesquisa da Faculdade Estácio de Goiás (DPE - Cl 013/2020).

\section{REFERÊNCIAS}

1. ASMAT U, et al. Diabetes mellitus and oxidative stress-A concise review. Saudi Pharm J, 2016; 24: 547-553.

2. BENEDICT C, et al. Intranasal insulin as a therapeutic option in the treatment of cognitive impairments. Exp. Gerontol, 2011; 46: 112-115.

3. BUTTERFIELD DA, et al. Elevated risk of type 2 diabetes for development of Alzheimer disease: a key role for oxidative stress in brain. Biochim. Biophys. Acta, 2014; 1842: 1693-1706.

4. CHAPMAN CD, et al. Intranasal insulin in Alzheimer's disease: Food for thought. Neuropharmacology, 2018; 136 : 196-201.

5. CHATTERJEE S, MUDHER A. Alzheimer's Disease and Type 2 Diabetes: A Critical Assessment of the Shared Pathological Traits. Front Neurosci, 2018; 12.

6. CHEN Z, ZHONG C. Decoding Alzheimer's disease from perturbed cerebral glucose metabolism: implications for diagnostic and therapeutic strategies. Prog. Neurobiol, 2013; 108: 21-43.

7. DIMEGLIO LA, et al. Type 1 diabetes. The Lancet, 2018; 391: 2449-2462.

8. FELICE FG, et al. How does brain insulin resistance develop in Alzheimer's disease? Alzheimers Dement, 2014; 10: S26-32.

9. FERREIRA ST, et al. Inflammation, defective insulin signaling, and neuronal dysfunction in Alzheimer's disease. Alzheimers Dement, 2014; 10: S76-83.

10. FRANKL JA, et al. T-cell receptor repertoire variation may be associated with type 2 diabetes mellitus in humans. Diabetes Metab. Res. Rev., 2016; 32: 297-307. 
11. GABBOUJ S, et al. Altered Insulin Signaling in Alzheimer's Disease Brain - Special Emphasis on PI3K-Akt Pathway. Front Neurosci, 2019; 13.

12. GOMES BF, ACCARDO CM. Immunoinflammatory mediators in the pathogenesis of diabetes mellitus. Einstein (São Paulo), 2019; 17.

13. HURRLE S, HSU WH. The etiology of oxidative stress in insulin resistance. Biomed J, 2017; 40: $257-262$.

14. KANDIMALLA R, et al. Is Alzheimer's disease a Type 3 Diabetes? A critical appraisal. Biochim Biophys Acta Mol Basis Dis, 2017; 1863: 1078-1089.

15. KLIP A, et al. Thirty sweet years of GLUT4. J. Biol. Chem., 2019; 294: 11369-11381.

16. LI R, et al. Effects and Underlying Mechanisms of Bioactive Compounds on Type 2 Diabetes Mellitus and Alzheimer's Disease. Oxid Med Cell Longev, 2019; 8165707.

17. LOCHHEAD JJ, et al. Distribution of insulin in trigeminal nerve and brain after intranasal administration. Scientific Reports, 2019; 9: 1-9.

18. MAGALHÃES FG, et al. Diabetes Tipo 1 e a Carreira Militar: uma revisão. RRS-FESGO, 2019; 02(3): $90-95$.

19. MEHTA RJ, et al. Mechanism of Action of Inhaled Insulin on Whole Body Glucose Metabolism in Subjects with Type 2 Diabetes Mellitus. Int J Mol Sci, 2019; 20.

20. MONTEIRO AMF, et al. Coping strategies among caregivers of people with Alzheimer disease: a systematic review. Trends Psychiatry Psychother, 2018; 40: 258-268.

21. NAZARETH AM. Type 2 diabetes mellitus in the pathophysiology of Alzheimer's disease. Dementia \& Neuropsychologia, 2017; 11: 105-113.

22. NETH BJ, CRAFT S. Insulin Resistance and Alzheimer's Disease: Bioenergetic Linkages. Front Aging Neurosci, 2017; 9.

23. NOBLE JA. Immunogenetics of type 1 diabetes: A comprehensive review. Journal of Autoimmunity, The Genetics of Autoimmunity, 2015; 64: 101-112.

24. OLIVEIRA JEP, et al. Diretrizes da Sociedade Brasileira de Diabetes 2017-2018. 2017. São Paulo: Editora Clannad; 2017.

25. PUGAZHENTHI S, et al. Common neurodegenerative pathways in obesity, diabetes, and Alzheimer's disease. Biochim Biophys Acta Mol Basis Dis, 2017; 1863: 1037-1045.

26. RAK K, BRONKOWSKA M. Immunomodulatory Effect of Vitamin D and Its Potential Role in the Prevention and Treatment of Type 1 Diabetes Mellitus-A Narrative Review. Molecules, 2018; 24.

27. RINALDI G, et al. Cost and cost-effectiveness of $m$ Health interventions for the prevention and control of type 2 diabetes mellitus: a protocol for a systematic review. BMJ Open, 2019; 9: e027490.

28. SANTIAGO JCP, HALLSCHMID M. Outcomes and clinical implications of intranasal insulin administration to the central nervous system. Exp. Neurol, 2019; 317: 180-190.

29. STRACHAN MW, et al. Is type II diabetes associated with an increased risk of cognitive dysfunction? A critical review of published studies. Diabetes Care, 1997; 20: 438-445.

30. SURKOVA EV, et al. Prospects of intranasal insulin for correction of cognitive impairments, in particular those associated with diabetes mellitus. Probl Endokrinol (Mosk), 2019; 65: 57-65.

31. TAOUIS M, TORRES-ALEMAN I. Editorial: Insulin and The Brain. Front Endocrinol (Lausanne), $2019 ; 10$.

32. THANARAJAH SE, et al. The role of insulin sensitivity and intranasally applied insulin on olfactory perception. Scientific Reports, 2019; 9: 1-8.

33. THOMAS NJ, et al. Frequency and phenotype of type 1 diabetes in the first six decades of life: a cross-sectional, genetically stratified survival analysis from UK Biobank. Lancet Diabetes Endocrinol, 2018; 6: 122-129.

34. VIEIRA MNN, et al. Connecting Alzheimer's disease to diabetes: Underlying mechanisms and potential therapeutic targets. Neuropharmacology, 2018; 136: 160-171.

35. WINGROVE J, et al. Characterization of nasal devices for delivery of insulin to the brain and evaluation in humans using functional magnetic resonance imaging. J Control Release, 2019; 302: 140-147. 